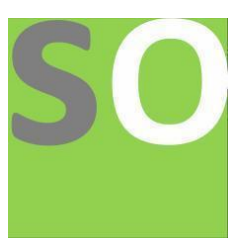

Article title: Traditional Chinese medicine combined with recombinant interferon to alleviate symptoms of COVID-19 infectious pneumonia

Authors: Yanchao Wang[1], Tao Liu[2], Weili Niu[3], Junzheng Yang[4]

Affiliations: Clinic laboratory of the third people's Hospital of Hebi[1], Guangdong nephrotic drug EngineeringTechnology Research Center, Guangdong Consun Pharmaceutical Group[2]

Orcid ids: 0000-0001-9992-3179[4]

Contact e-mail: yangjunzheng606403@163.com

License information: This work has been published open access under Creative Commons Attribution License http://creativecommons.org/licenses/by/4.0/, which permits unrestricted use, distribution, and reproduction in any medium, provided the original work is properly cited. Conditions, terms of use and publishing policy can be found at https://www.scienceopen.com/.

Preprint statement: This article is a preprint and has not been peer-reviewed, under consideration and submitted to ScienceOpen Preprints for open peer review.

Funder: None

DOI: 10.14293/S2199-1006.1.SOR-.PPOVB2Y.v1

Preprint first posted online: 18 January 2022

Keywords: COVID-19, recombinant interferon, Traditional Chinese medicine, Lopinavir/Ritonavir, Corona virus 


\title{
Traditional Chinese medicine combined with recombinant interferon to alleviate symptoms of
}

\section{COVID-19 infectious pneumonia}

\author{
Yanchao Wang ${ }^{1}$, Tao $\mathrm{Liu}^{1}$, Weili Niu${ }^{1}$, Junzheng Yang ${ }^{2 *}$ \\ ${ }^{1}$ Clinic laboratory of the third people's Hospital of Hebi, Hebi, Henan 458000, People's Republic of China; \\ ${ }^{2}$ Guangdong nephrotic drug EngineeringTechnology Research Center, Institute of Consun Co. for Chinese Medicine in Kidney \\ Diseases, Guangdong Consun Pharmaceutical Group, Guangzhou 510530, \\ People's Republic of China
}

*Correspondence to Junzheng Yang (yangjunzheng606403@163.com), Guangdong nephrotic drug EngineeringTechnology Research Center, Institute of Consun Co.

for Chinese Medicine in Kidney Diseases, Guangdong Consun Pharmaceutical Group, Guangzhou 510530, People’s Republic of China.

\begin{abstract}
Objectives: Collect and analyze the basic information, symptoms, therapeutic method and therapeutic effect of novel coronavirus (COVID-19) infected pneumonia patients in the hospital in northern Henan Province from January 2020 to April 2020, to provide evidences for prevention and treatment of COVID-19 infectious pneumonia. Methods: The information of patients of COVID-19 infectious pneumonia in the hospital were collected and analyzed, including the admission time, the discharge time, therapeutic method, and therapeutic effect after treatment.

Results: A total of 10 cases patients of COVID-19 infectious pneumonia were collected, there were 5 male patients and 5 female patients, the age ranged from 16-54 years old and the average age was 34.2 years old; the body temperature of 6 cases patients were higher than $37^{\circ} \mathrm{C}$ when they were admitted to hospital; CT results showed that all the patients had infectious lesions of both lungs, and the results of nucleic acid test were positive. 5 million (aerosol inhalation) recombinant interferon (Lopinavir/Ritonavir or Lopinavir/Ritonavir/Abidol) combined with
\end{abstract}


traditional Chinese medicine could alleviate effectively symptoms of COVID-19 infectious pneumonia within a month, the body temperature of patients returned to normal, respiratory symptoms improved significantly, COVID-19 nucleic acid test was negative, and results of lung imaging was also normal. Conclusion: Lopinavir/Ritonavir or Lopinavir/Ritonavir/Abidol combined with Traditional Chinese medicine has a good effect on relieving the symptoms of COVID-19 infected pneumonia and has significance for clinical treatment or clinic remission of COVID-19 infectious pneumonia.

Keywords: COVID-19; recombinant interferon; Traditional Chinese medicine; Lopinavir/Ritonavir.

\section{Introduction}

COVID-19 infectious pneumonia has been rapidly spread from Wuhan city to the whole China and to every corner of the world since it was discovered in Wuhan from December 2019. So far, it has mutated several times, which has seriously affected the daily life and health of people in the world, and the number of infections has reached the highest in history ${ }^{[1,2]}$. COVID-19 infectious pneumonia is characterized by high pathogenicity, spreads rapidly and is prone to mutation. The infected patients often have higher body temperature, fever, dry cough, fatigue; CT results show pulmonary infectious lesions and the results of nucleic acid tests are positive ${ }^{[3,4,5,6]}$. So far, there is no ideal therapeutic methods for COVID-19 infectious pneumonia treatment.

The clinical drugs of COVID-19 infectious pneumonia have been reported mainly include protease inhibitors (ritonavir, oseltamivir, darunavir, and lopinavir $)^{[7,8]}$, RNA-dependent RNA polymerase ${ }^{[9,10,11,12]}$, these drugs have various side effects in the treatment of COVID-19 patients $^{[13]}$, or the therapeutic effect is poor when used alone or in combination with two kind of different drugs ${ }^{[14]}$. For this purpose, This article provided a novel treatment through summarizing the data of 10 COVID-19 patients who came to our hospital in North Henan Province, 
collecting and analyzing the basic information and clinical treatment, and found that when using Lopinavir/Ritonavir or Lopinavir/Ritonavir/Abidol interferon, combination of traditional Chinese medicine had better therapeutic effect in the treatment of COVID-19 infectious pneumonia.

\section{General information of patients}

A total of 10 effective COVID-19 patients were collected in this report. There were 5 males and 5 females, aged from 16-54 years old, and the average age was 34.2 years old; there were 8 cases of patients with Wuhan contact history, after using Lopinavir/Ritonavir or Lopinavir/Ritonavir/Abidol interferon, combination of Chinese traditional medicine, the symptoms of COVID-19 infectious pneumonia disappeared within one month, the detail showed at Table 1.

Table 1. Summary the basic information of 10 cases of COVID-19 patients in North Henan Province

\begin{tabular}{ccccccc}
\hline Patients & Gender & Age & $\begin{array}{l}\text { Is there any contact } \\
\text { history in Wuhan }\end{array}$ & Admission time & Discharge time & Treatment period \\
\hline 1 & Male & 25 & Yes & 01.27 .2020 & 02.16 .2020 & 17 days \\
2 & Female & 54 & Yes & 02.08 .2020 & 02.29 .2020 & 21 days \\
3 & Female & 16 & Yes & 01.26 .2020 & 02.12 .2020 & 15 days \\
4 & Male & 32 & Unknown & 02.06 .2020 & 02.26 .2020 & 20 days \\
5 & Male & 54 & Yes & 02.08 .2020 & 02.29 .2020 & 21 days \\
6 & Male & 34 & No(Shanghai) & 02.01 .2020 & 02.19 .2020 & 18 days \\
7 & Male & 31 & Yes & 02.07 .2020 & 02.26 .2020 & 19 days
\end{tabular}




\begin{tabular}{cccccccc}
8 & Female & 42 & Yes & 01.25 .2020 & 02.10 .2020 & 14 days \\
9 & Female & 23 & Yes & 02.09 .2020 & 02.28 .2020 & 19 days \\
10 & Female & 31 & Yes & 02.06 .2020 & 02.28 .2020 & 22 days \\
\hline
\end{tabular}

\section{Therapeutic method}

10 cases of COVID-19 infectious pneumonia patients were treated with Lopinavir/Ritonavir or Lopinavir/Ritonavir/Abidol combined with traditional Chinese medicine to relieve symptoms. The main treatment process was to inject 5 million units of $r$ Lopinavir/Ritonavir or Lopinavir/Ritonavir/Abidol to inhibit COVID-19 virus, combined with various traditional Chinese medicines to improve the immunity of COVID-19 patients or relieve the symptoms of COVID-19 patients, and every patient had different kinds of traditional Chinese medicines for use (Table 2).

Table 2. Summary of treatment methods for 10 cases of COVID-19 patients in North Henan Province

\begin{tabular}{ccc}
\hline Patients & Recombinant interferon & Traditional Chinese medicine \\
\hline & & Raw honeysuckle, Forsythia, Platycodon grandiflorum, Bamboo shavings, White \\
& Leony, Mulberry leaf, Chrysanthemum, Platycodon grandiflorum, Licorice, \\
& & Forsythia, Atractylodes rhizome, Earthworm, Amomum villosum, Ophiopogon \\
& japonicus, Fried hawthorn, Poria cocos and Ginger \\
\hline & Lopinavir/Ritonavir & Cinnamon alisma orientalis, Poria cocos, Atractylodes macrocephala, Ginger, \\
& Pinellia, Magnolia obavata, Fried almond, Cangerine peel, Patchouli, Grass fruit, \\
\hline
\end{tabular}


Atractylodes macrocephala, Platycodon grandiflorum, Fried Scutellaria and

Forsythia suspensa

\begin{tabular}{cr}
\hline Lopinavir/Ritonavir & $\begin{array}{c}\text { Forsythia suspensa } \\
\text { Rhizoma atractylodis, Honeysuckle, Mint, Scutellaria baicalensis, Patchouli, Poria } \\
\text { cocos, Coptis chinensis, Grass fruit, Licorice and Amomum villosum }\end{array}$ \\
\hline 4 & Scutellaria baicalensis, Coptis chinensis, Forsythia suspensa, Honeysuckle, Yunling, \\
& Huoxiang, Perilla, Alisma tulip, Stemona root, Almond, Hemp, Perilla, Chaihu, \\
& Guanzhong, Gypsum, Honeysuckle, Sea cucumber, astragalus, Radix scrophulariae, \\
& \\
\hline
\end{tabular}

Fried Scutellaria, Almond, Atractylodes macrocephala,

Yunling, Mulberry leaves, Stemona root, Astragalus root,

$5 \quad$ Lopinavir/Ritonavir/Abidol

Angelica root, Notopterygium root, Licorice root,

Scutellaria root, Forsythia root, Honeysuckle, Alisma orientalis,

Sea cucumber, Astragalus root, Yujin, Radix scrophulariae

and Licorice root

Raw honeysuckle, Scutellaria baicalensis, Peppermint, Mulberry leaf, 
Stemona, Red patchouli, Grass fruit, Tulip, Bombyx mori, Perilla, Scorched

hawthorn, Licorice, Ginger

\begin{tabular}{ccc}
\hline 7 & Lopinavir/Ritonavir/Abidol & hawthorn, Licorice, Ginger \\
\hline 8 & Lopinavir/Ritonavir/Abidol & Radix rehmanniae, Poria cocos, Honeysuckle, Scutellaria and Atractylodes \\
\hline 9 & Lopinavir/Ritonavir/Abidol & Scutellaria, Honeysuckle, Forsythia, Hypericum, \\
& & Radix Scrophulariae, Rhizoma Coptidis, Radix Pseudostellariae, Licorice and \\
\hline 10 & Lopinavir/Ritonavir/Abidol & Maxing Shigan Decoction and Xiaochaihu Decoction
\end{tabular}

\section{Therapeutic effect}

10 cases of COVID-19 infectious pneumonia patients were treated with Lopinavir/Ritonavir or Lopinavir/Ritonavir/Abidol combined with Traditional Chinese medicine. fever and basic symptoms of COVID-19 infectious pneumonia disappeared, the body temperature returned to normal, CT results was normal, and nucleic acid detection was negative for two times, but detection of WBC (white blood cell), RBC (Red blood cell), Hemoglobin (HGB) and platelet (PLT) were no obvious changes before treatment and after treatment (Table 3).

Table 3. Comparison of symptoms of 10 cases of New COVID-19 infected pneumonia patients before and after treatment

\begin{tabular}{lcccc}
\hline Body & Symptom & CT detection & WBC & Nucleic \\
temperature & & HGB test & RBC \\
\hline
\end{tabular}




\begin{tabular}{|c|c|c|c|c|c|c|c|c|c|}
\hline \multirow{2}{*}{1} & $\begin{array}{l}\text { Before } \\
\text { treatment }\end{array}$ & $37.2^{\circ} \mathrm{C}$ & $\begin{array}{l}\text { Fever with } \\
\text { retching } \\
\text { for } 2 \text { days }\end{array}$ & $\begin{array}{l}\text { Obsolete lesions of } \\
\text { upper lobes in both } \\
\text { lungs }\end{array}$ & $\begin{array}{c}4.64 \times 10^{9} / \mathrm{L}\left(\mathrm{N}: 3.15 \times 10^{9} / \mathrm{L}\right. \\
\mathrm{L}: 1.02 \times 10^{9} / \mathrm{L} \\
\mathrm{N}: 67.9 \%, \mathrm{~L}: 22 \%)\end{array}$ & $5.21 \times 10^{12} / \mathrm{L}$ & $130 \mathrm{~g} / \mathrm{L}$ & $176 \times 10^{9} / \mathrm{L}$ & Positive \\
\hline & $\begin{array}{c}\text { After } \\
\text { treatment }\end{array}$ & Normal & $\begin{array}{l}\text { Symptom } \\
\text { disappeared }\end{array}$ & $\begin{array}{l}\text { No obvious } \\
\text { abnormality }\end{array}$ & $\begin{array}{c}5.49 \times 10^{9} / \mathrm{L} \\
\left(\mathrm{N}: 3.18 \times 10^{9} / \mathrm{L} ; \mathrm{L}: 1.91 \times 10^{9} / \mathrm{L} ;\right. \\
\mathrm{N}: 58 \%, \mathrm{~L}: 34.8 \%)\end{array}$ & $4.91 \times 10^{12} / \mathrm{L}$ & $145 \mathrm{~g} / \mathrm{L}$ & $204 \times 10^{9} / \mathrm{L}$ & Negative \\
\hline \multirow{2}{*}{2} & $\begin{array}{l}\text { Before } \\
\text { treatment }\end{array}$ & $37.1^{\circ} \mathrm{C}$ & $\begin{array}{l}\text { Cough and } \\
\text { sputum, fever }\end{array}$ & $\begin{array}{c}\text { Infectious lesions } \\
\text { of both lungs }\end{array}$ & $\begin{array}{c}5.0 \times 10^{9} / \mathrm{L} \\
\left(\mathrm{N}: 32.97 \times 10^{9} / \mathrm{L},\right. \\
\mathrm{L}: 1.81 \times 10^{9} / \mathrm{L} \\
\mathrm{N}: 59.4 \%, \mathrm{~L}: 36.2 \%)\end{array}$ & $4.39 \times 10^{12} / \mathrm{L}$ & Unknown & Unknown & Positive \\
\hline & $\begin{array}{l}\text { After } \\
\text { treatment }\end{array}$ & Normal & $\begin{array}{l}\text { Respiratory } \\
\text { symptoms } \\
\text { disappeared }\end{array}$ & $\begin{array}{l}\text { No obvious } \\
\text { abnormality }\end{array}$ & $\begin{array}{c}3.73 \times 10^{9} / \mathrm{L} \\
\left(\mathrm{N}: 1.78 \times 10^{9} / \mathrm{L},\right. \\
\mathrm{L}: 1.64 \times 109 / \mathrm{L} ; \mathrm{N}: 47.8 \% \\
\mathrm{~L}: 44 \%)\end{array}$ & $3.75 \times 10^{12} / \mathrm{L}$ & $122 \mathrm{~g} / \mathrm{L}$ & $159 \times 10^{9} / \mathrm{L}$ & Negative \\
\hline \multirow[b]{2}{*}{3} & $\begin{array}{l}\text { Before } \\
\text { treatment }\end{array}$ & $37.6^{\circ} \mathrm{C}$ & $\begin{array}{c}\text { Headache and } \\
\text { fever }\end{array}$ & $\begin{array}{l}\text { Infectious lesion of } \\
\text { right lower lobe of } \\
\text { lung }\end{array}$ & $\begin{array}{c}3.59 \times 10^{9} / \mathrm{L} \\
\left(\mathrm{N}: 1.6 \times 10^{9} / \mathrm{L}\right. \\
\mathrm{L}: 1.59 \times 10^{9} / \mathrm{L} \\
\mathrm{N}: 46.83 \% \mathrm{~L}: 44.83 \%)\end{array}$ & $4.67 \times 10^{12} / \mathrm{L}$ & $133 \mathrm{~g} / \mathrm{L}$ & $212 \times 10^{9} / \mathrm{L}$ & Positive \\
\hline & $\begin{array}{l}\text { After } \\
\text { treatment }\end{array}$ & Normal & $\begin{array}{l}\text { Symptom } \\
\text { disappeared }\end{array}$ & $\begin{array}{l}\text { No obvious } \\
\text { abnormality }\end{array}$ & $\begin{array}{c}4.07 \times 10^{9} / \mathrm{L} \\
\left(\mathrm{N}: 1.61 \times 10^{9} / \mathrm{L}\right. \\
\mathrm{L}: 2.11 \times 10^{9} / \mathrm{L} ; \mathrm{N}: 39.7 \% \\
\mathrm{~L}: 52 \%)\end{array}$ & $4.98 \times 10^{12} / \mathrm{L}$ & $147 \mathrm{~g} / \mathrm{L}$ & $238 \times 10^{9} / \mathrm{L}$ & Negative \\
\hline 4 & $\begin{array}{l}\text { Before } \\
\text { treatment }\end{array}$ & $37.3^{\circ} \mathrm{C}$ & $\begin{array}{l}\text { Cough and } \\
\text { sputum, fever }\end{array}$ & $\begin{array}{l}\text { Infectious lesions } \\
\text { of both lungs }\end{array}$ & $\begin{array}{c}4.52 \times 10^{9} / \mathrm{L} \\
\left(\mathrm{N}: 2.11 \times 10^{9} / \mathrm{L}\right. \\
\mathrm{L}: 1.8 \times 10^{9} / \mathrm{L} ; \mathrm{N}: 46.7 \% \\
\mathrm{~L}: 39.9 \%)\end{array}$ & $5.03 \times 10^{12} / \mathrm{L}$ & I & $176 \times 10^{9} / \mathrm{L}$ & Positive \\
\hline
\end{tabular}




\begin{tabular}{|c|c|c|c|c|c|c|c|c|c|}
\hline & $\begin{array}{c}\text { After } \\
\text { treatment }\end{array}$ & Normal & $\begin{array}{c}\text { Symptom } \\
\text { disappeared }\end{array}$ & $\begin{array}{l}\text { The pulmonary } \\
\text { lesions were } \\
\text { slightly absorbed } \\
\text { than before }\end{array}$ & $\begin{array}{c}4.07 \times 10^{9} / \mathrm{L} \\
\left(\mathrm{N}: 1.61 \times 10^{9} / \mathrm{L}\right. \\
\text { L: } 2.11 \times 10^{9} / \mathrm{L} ; \\
\mathrm{N}: 39.7 \%, \mathrm{~L}: 52 \%)\end{array}$ & $4.98 \times 10^{12} / \mathrm{L}$ & 147g/L & $238 \times 10^{9} / \mathrm{L}$ & Negative \\
\hline & $\begin{array}{l}\text { Before } \\
\text { treatment }\end{array}$ & Normal & No symptom & $\begin{array}{l}\text { Infectious lesions } \\
\text { of both lungs }\end{array}$ & $\begin{array}{c}7.88 \times 10^{9} / \mathrm{L} \\
\left(\mathrm{N}: 6.42 \times 10^{9} / \mathrm{L}\right. \\
\mathrm{L}: 1.11 \times 10^{9} / \mathrm{L}, \mathrm{N}: 81.4 \% \\
\mathrm{~L}: 14.2 \%)\end{array}$ & $5.28 \times 10^{12} / \mathrm{L}$ & I & $183 \times 10^{9} / \mathrm{L}$ & Positive \\
\hline 5 & $\begin{array}{c}\text { After } \\
\text { treatment }\end{array}$ & Normal & No symptom & $\begin{array}{l}\text { The pulmonary } \\
\text { lesions were } \\
\text { slightly absorbed } \\
\text { than before }\end{array}$ & $\begin{array}{c}3.75 \times 10^{9} / \mathrm{L} \\
\left(\mathrm{N}: 2.2 \times 10^{9} / \mathrm{L},\right. \\
\text { L:1.164×10 } \\
\text { L: } 31 \%)\end{array}$ & $5.15 \times 10^{12} / \mathrm{L}$ & 149g/L & $154 \times 10^{9} / \mathrm{L}$ & Negative \\
\hline \multirow[t]{2}{*}{6} & $\begin{array}{l}\text { Before } \\
\text { treatment }\end{array}$ & $38^{\circ} \mathrm{C}$ & $\begin{array}{c}\text { Fever, cough } \\
\text { with a small } \\
\text { amount of white } \\
\text { phlegm }\end{array}$ & $\begin{array}{l}\text { Infectious lesions } \\
\text { of both lungs }\end{array}$ & $\begin{array}{c}6.98 \times 10^{9} / \mathrm{L} \\
\left(\mathrm{N}: 4.36 \times 10^{9} / \mathrm{L}, \mathrm{N}: 62.4 \%\right. \\
\text { L: } 27.7 \%)\end{array}$ & $5.31 \times 10^{12} / \mathrm{L}$ & 153g/L & $177 \times 10^{9} / \mathrm{L}$ & Positive \\
\hline & $\begin{array}{c}\text { After } \\
\text { treatment }\end{array}$ & Normal & $\begin{array}{c}\text { Symptom } \\
\text { disappeared }\end{array}$ & $\begin{array}{l}\text { No obvious } \\
\text { abnormality }\end{array}$ & $\begin{array}{c}6.25 \times 10^{9} / \mathrm{L}\left(\mathrm{N}: 3.98 \times 10^{9} / \mathrm{L}\right. \\
\text { L: } 1.42 \times 10^{9} / \mathrm{L} ; \mathrm{N}: 63.7 \%, \\
\text { L: } 22.8 \%)\end{array}$ & $4.72 \times 10^{12} / \mathrm{L}$ & 132g/L & $268 \times 10^{9} / \mathrm{L}$ & Negative \\
\hline \multirow[t]{2}{*}{7} & $\begin{array}{l}\text { Before } \\
\text { treatment }\end{array}$ & $36^{\circ} \mathrm{C}$ & Fever & $\begin{array}{l}\text { Patchy shadow of } \\
\text { right upper lung } \\
\text { and lower lobe of } \\
\text { both lungs }\end{array}$ & $\begin{array}{c}3.28 \times 10^{9} / \mathrm{L}\left(\mathrm{N}: 1.40 \times 10^{9} / \mathrm{L}\right. \\
\mathrm{L}: 1.59 \times 10^{9} / \mathrm{L} \\
\mathrm{N}: 42.72 \%, \mathrm{~L}: 48.47 \%)\end{array}$ & $5.30 \times 10^{12} / \mathrm{L}$ & 155g/L & $107 \times 10^{9} / \mathrm{L}$ & Positive \\
\hline & $\begin{array}{c}\text { After } \\
\text { treatment }\end{array}$ & Normal & $\begin{array}{c}\text { Symptom } \\
\text { disappeared }\end{array}$ & $\begin{array}{l}\text { No obvious } \\
\text { abnormality }\end{array}$ & $\begin{array}{c}6.28 \times 10^{9} / \mathrm{L} \\
\left(\mathrm{N}: 3.30 \times 10^{9} / \mathrm{L}\right. \\
\mathrm{L}: 2.52 \times 10^{9} / \mathrm{L} ; \mathrm{N}: 52.6 \%\end{array}$ & $5.16 \times 10^{12} / \mathrm{L}$ & 152g/L & $195 \times 10^{9} / \mathrm{L}$ & Negative \\
\hline
\end{tabular}




\begin{tabular}{|c|c|c|c|c|c|c|c|c|c|}
\hline \multirow{2}{*}{8} & $\begin{array}{l}\text { Before } \\
\text { treatment }\end{array}$ & $38.1^{\circ} \mathrm{C}$ & $\begin{array}{c}\text { Cough and } \\
\text { sputum, fever }\end{array}$ & $\begin{array}{l}\text { Infectious lesions } \\
\text { of both lungs }\end{array}$ & $\begin{array}{c}3.7 \times 10^{9} / \mathrm{L} \\
\left(\mathrm{N}: 2.51 \times 10^{9} / \mathrm{L},\right. \\
\mathrm{L}: 0.94 \times 10^{9} / \mathrm{L} ; \\
\mathrm{N}: 67.8 \%, \mathrm{~L}: 25.6 \%)\end{array}$ & $4.03 \times 10^{12} / \mathrm{L}$ & $122 \mathrm{~g} / \mathrm{L}$ & $166 \times 10^{9} / \mathrm{L}$ & Positive \\
\hline & $\begin{array}{c}\text { After } \\
\text { treatment }\end{array}$ & Normal & $\begin{array}{l}\text { Symptom } \\
\text { disappeared }\end{array}$ & $\begin{array}{l}\text { No obvious } \\
\text { abnormality }\end{array}$ & $\begin{array}{c}5.23 \times 10^{9} / \mathrm{L} \\
\left(\mathrm{N}: 2.84 \times 10^{9} / \mathrm{L},\right. \\
\mathrm{L}: 1.91 \times 10^{9} / \mathrm{L} ; \mathrm{N}: 54.3 \%, \\
\mathrm{~L}: 36.6 \%)\end{array}$ & $3.9 \times 10^{12} / \mathrm{L}$ & $118 \mathrm{~g} / \mathrm{L}$ & $338 \times 10^{9} / \mathrm{L}$ & Negative \\
\hline \multirow{2}{*}{9} & $\begin{array}{l}\text { Before } \\
\text { treatment }\end{array}$ & 1 & $\begin{array}{l}\text { Cough, fatigue } \\
\text { and poor } \\
\text { appetite }\end{array}$ & $\begin{array}{c}\text { Infectious lesions } \\
\text { of lower lobes of } \\
\text { both lungs }\end{array}$ & $\begin{array}{c}3.93 \times 10^{9} / \mathrm{L} \\
\left(\mathrm{N}: 1.29 \times 10^{9} / \mathrm{L},\right. \\
\mathrm{L}: 2.24 \times 10^{9} / \mathrm{L} ; \mathrm{N}: 33.0 \%, \\
\mathrm{~L}: 56.6 \%)\end{array}$ & $4.37 \times 10^{12} / \mathrm{L}$ & $130 \mathrm{~g} / \mathrm{L}$ & $255 \times 10^{9} / \mathrm{L}$ & Positive \\
\hline & $\begin{array}{l}\text { After } \\
\text { treatment }\end{array}$ & Normal & $\begin{array}{c}\text { Symptom } \\
\text { disappeared }\end{array}$ & $\begin{array}{l}\text { No obvious } \\
\text { abnormality }\end{array}$ & $\begin{array}{c}4.9 \times 10^{9} / \mathrm{L} \\
\left(\mathrm{N}: 2.34 \times 10^{9} / \mathrm{L}\right. \\
\mathrm{L}: 2.08 \times 10^{9} / \mathrm{L} ; \mathrm{N}: 47.8 \%, \\
\mathrm{~L}: 42.6 \%)\end{array}$ & $3.87 \times 10^{12} / \mathrm{L}$ & $121 \mathrm{~g} / \mathrm{L}$ & $289 \times 10^{9} / \mathrm{L}$ & Negative \\
\hline \multirow{2}{*}{10} & $\begin{array}{l}\text { Before } \\
\text { treatment }\end{array}$ & $38.6^{\circ} \mathrm{C}$ & Fever & $\begin{array}{l}\text { Infectious lesion of } \\
\text { right lower lobe of } \\
\text { lung }\end{array}$ & $\begin{array}{c}4.39 \times 10^{9} / \mathrm{L} \\
\left(\mathrm{N}: 2.41 \times 10^{9} / \mathrm{L}, \mathrm{L}:\right. \\
1.68 \times 10^{9} / \mathrm{L} ; \mathrm{N}: 54.89 \% \\
\mathrm{~L}: 38.4 \%)\end{array}$ & $4.12 \times 10^{12} / \mathrm{L}$ & $121 \mathrm{~g} / \mathrm{L}$ & $190 \times 10^{9} / \mathrm{L}$ & Positive \\
\hline & $\begin{array}{c}\text { After } \\
\text { treatment }\end{array}$ & Normal & $\begin{array}{c}\text { Symptom } \\
\text { disappeared }\end{array}$ & $\begin{array}{l}\text { No obvious } \\
\text { abnormality }\end{array}$ & $\begin{array}{c}8.27 \times 10^{9} / \mathrm{L} \\
\left(\mathrm{N}: 4.93 \times 10^{9} / \mathrm{L}\right. \\
\mathrm{L}: 2.72 \times 109 / \mathrm{L} ; \mathrm{N}: 59.6 \%, \\
\mathrm{~L}: 32.89 \%)\end{array}$ & $4.44 \times 10^{12} / \mathrm{L}$ & $125 \mathrm{~g} / \mathrm{L}$ & $512 \times 10^{9} / \mathrm{L}$ & Negative \\
\hline
\end{tabular}




\section{Discussion}

This article collected and analyzed 10 cases of COVID-19 infectious pneumonia patients in North Henan Province. The basic information and therapeutic effect before and after treatment were sorted and analyzed. It was found that Lopinavir/Ritonavir or Lopinavir/Ritonavir/Abidol combined with Traditional Chinese medicine could eliminate symptoms of COVID-19 infected pneumonia in a shorter time, and nucleic acid detection is negative, which has good clinical significance.

We found a 16-year-old COVID-19 infectious pneumonia patients, which was youngest patient in this investigation. After Lopinavir/Ritonavir combined with Traditional Chinese medicine (Rhizoma atractylodis, Honeysuckle, Mint, Scutellaria baicalensis, Patchouli, Poria cocos, Coptis chinensis, Grass fruit, Licorice and Amomum villosum) treatment, the symptoms disappeared and nucleic acid test was negative within 15 days. Those data may provide a reference treatment for teenagers because of particularity of population.

It should be noted that Lopinavir/Ritonavir or Lopinavir/Ritonavir/Abidol combined with traditional Chinese medicine can eliminate symptoms of COVID-19 infected pneumonia and negative nucleic acid test. However, there are no significant changes in WBC, RBC, HGB and PLT before and after treatment. Therefore, WBC, RBC, HGB and PLT cannot not be used as a clinical index for COVID-19 infectious pneumonia. On the contrary, fever, CT detection and nucleic acid changed significantly before and after treatment, which has good clinical guiding significance.

The limitation of this article: 1. the patients in this investigation were few, and most of them come from northern Henan Province; 2. there are many kinds of traditional Chinese medicine used, so it is difficult to define which traditional Chinese medicine components work and how to 
work.

\section{Conflict of interests}

The authors declare that they have no competing interests in this article.

\section{Acknowledgements}

\section{None.}

\section{Funding}

None.

\section{References}

[1] Wuhan Municipal Health Commission. Report of clustering pneumonia of unknown etiology in Wuhan City. Published December 31, 2019. http://wjw.wuhan.gov.cn/front/web/showDetail/2019123108989.

[2] W.H. Organization. Coronavirus Disease 2019 (COVID-19): Situation Report 76. 2020, April 5.

[3] Chaolin Huang, Yeming Wang, Xingwang Li, Lili Ren, Jianping Zhao, Yi Hu, Li Zhang, et al. Clinical features of patients infected with 2019 novel coronavirus in Wuhan, China [J]. Lancet, 2020, 395(10223): 497-506.

[4] Jun Zheng. SARS-CoV-2: an Emerging Coronavirus that Causes a Global Threat. Int J Biol Sci [J]. 2020, 16(10): 1678-1685.

[5] Chen N, Zhou M, Dong X, Qu J, Gong F, Han Y. et al. Epidemiological and clinical characteristics of 99 cases of 2019 novel coronavirus pneumonia in Wuhan, China: a descriptive study [J].Lancet,2020, 395:507-513.

[6] Guan WJ, Ni ZY, Hu Y, Liang WH, Ou CQ, He JX. et al. Clinical characteristics of coronavirus disease 2019 in China[J]. N Engl J Med., 2020. 
[7] Kim J.Y. Letter to the editor: case of the index patient who caused tertiary transmission of coronavirus disease 2019 in Korea: the application of lopinavir/ritonavir for the treatment of COVID-19 pneumonia monitored by quantitative RT-PCR[J]. J. Korean Med. Sci., 2020, $35(7):$ e88.

[8] Cao B., Wang Y., Wen D., Liu W., Wang J., Fan G. et al. A trial of lopinavir-ritonavir in adults hospitalized with severe Covid-19[J]. N. Engl. J. Med., 2020, 382:1787-1799.

[9] Lim J., Jeon S., Shin H.Y., Kim M.J., Seong Y.M., Lee W.J., Choe K.W., et al. Case of the index patient who caused tertiary transmission of COVID-19 infection in Korea: the application of lopinavir/ritonavir for the treatment of COVID-19 infected pneumonia monitored by quantitative RT-PCR[J]. J. Korean Med. Sci., 2020, 35(6):e79.

[10] Guo Y.R., Cao Q.D., Hong Z.S., Tan Y.Y., Chen S.D., Jin H.J., Tan K.S., Wang D.Y., Yan Y. The origin, transmission and clinical therapies on coronavirus disease 2019 (COVID-19) outbreak -an update on the status[J]. Mil Med Res., 2020;7(1):11.

[11] Lung J., Lin Y.-S., Yang Y.-H., Chou Y.-L., Shu L.-H., Cheng Y.-C., Liu H.T., Wu C.-Y. The potential chemical structure of anti-SARS-CoV-2 RNA-dependent RNA polymerase[J]. J. Med. Virol., 2020, 92: 693-697.

[12] De Clercq E. New nucleoside analogues for the treatment of hemorrhagic fever virus infections[J]. Chem. Asian J., 2019, 14(22): 3962-3968.

[13] Li G., De Clercq E. Therapeutic options for the 2019 novel coronavirus (2019-nCoV) [J]. Nat. Rev. Drug Discov., 2020, 19(3):149-150.

[14] Bin Cao, Yeming Wang, Danning Wen, et al. A Trial of Lopinavir-Ritonavir in Adults Hospitalized with Severe Covid-19[J]. N Engl J Med., 2020: NEJMoa2001282.

[15] Ivan Fan-Ngai Hung, Kwok-Cheung Lung, Eugene Yuk-Keung Tso, et al. Triple combination of interferon beta-1b, lopinavir-ritonavir, and ribavirin in the treatment of patients admitted to hospital with COVID-19: an open-label, randomised, phase 2 trial[J]. Lancet, 2020, 395(10238): 1695-1704. 
